\title{
Statistical Analysis to find an impact of temperature on novel coronavirus (COVID-19)
}

CHESTA DHINGRA, M.Sc. Information Technology, Certified Predictive Business Analyst

Research Data Analyst, Sanrachna, Shree Guru Gobind Singh Tricentenary University

Correspondence: chesta_dhingra@sgtuniversity.org, Tel: +918800788019

\begin{abstract}
The study tries to explain the impact of high temperature on the number of confirmed cases by excluding all measures that have been taken by specific governments in certain global hotspots like New York City, Lombardy, Madrid (Spain) and Maharashtra. Some statistical analysis like F-statistical test, ANOVA and t-test have been performed to know how much variance is there among the regions for the parameters 1) proportion of cumulative confirmed cases to the whole population and 2) mean temperature. The analysis includes graphs to have a clear visualization at a first glance for all four zones that have been taken for case study and ends with the all statistical results, discussion and conclusion of the tests that have been performed.
\end{abstract}

Keywords: f-statistical test, ANOVA (Analysis of Variance), t-test, temperature, Coronavirus, Pearson's Correlation

\section{Introduction}

The outbreak of this novel SARS-CoV-2 overwhelmed the global health system making an outrageous mark on each and every aspect of life be it social, economic and psychological. All around the world researchers and scientists are trying to solve the mystery or questions behind the spread of this virus which is highly contagious looking at all those parameters which can help in curbing the spread as, till now it has affected almost 4,553,432 people globally. In this particular paper we are looking at those parts of the world which have been highly impacted by this pandemic and trying to relate $\&$ analyse how much temperature as a parameter can help in controlling the spread. The data has been looked from $10^{\text {th }}$ March 2020 onwards till $23^{\text {rd }}$ April 2020, New York has been the worst hit by this pandemic till $23^{\text {rd }}$ April 2020 having 1,45,855 number of confirmed cases, on second Lombardy with number of confirmed cases 70,165, third is Madrid with 61,588 and lastly Maharashtra with 5652.
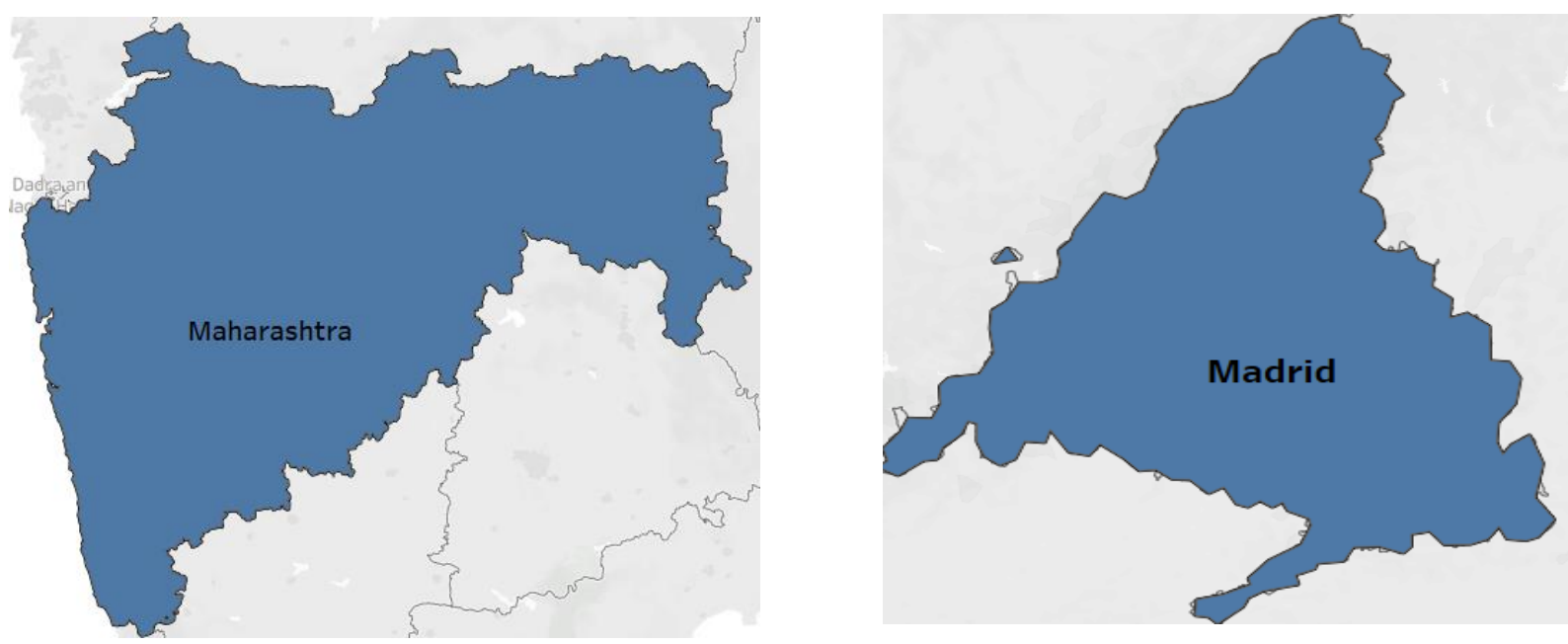

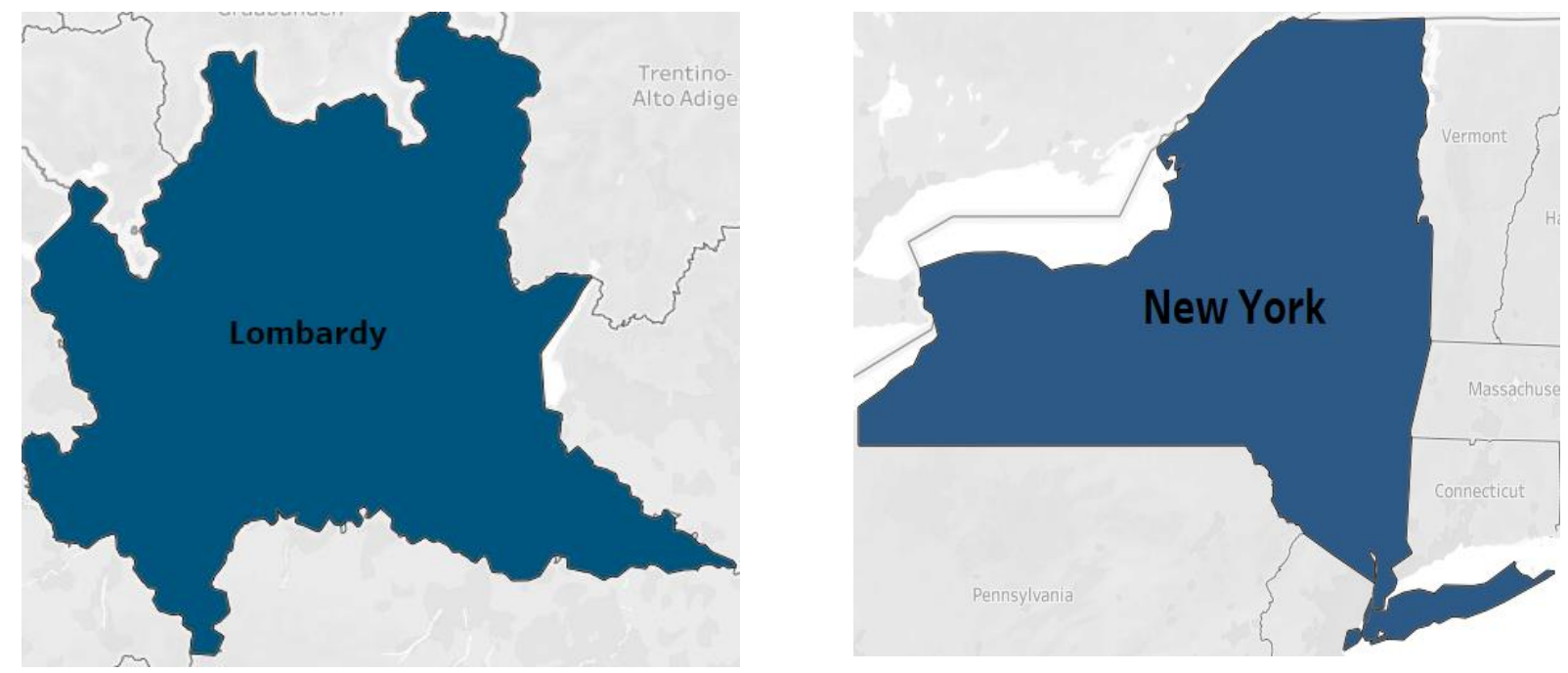

The aim is to look for how much variance is there in number of confirmed cases and the variance in the temperature, thus having similarity or less variance in the temperature parameter can relate to have less variance among the number of confirmed cases in all these four zones that have been taken into consideration for the case study.

\section{Materials and Methods}

Data collection for confirmed COVID-19 cases for all the zones have been taken from Wikipedia site and the temperature data has been taken on hourly basis from the IBM weather data as per the longitude \& latitude of the concerned regions from $10^{\text {th }}$ March 2020 to $23^{\text {rd }}$ April 2020.

To have certain view regarding the relationship between the two variables we get the cumulative proportion value of the confirmed cases to the whole population and the average or mean temperature. With the help of graphical representations, we'll able to visualize that area with relatively high temperature has comparatively less cases as compared to areas with low temperature which is going maximum till 18 degree Celsius.

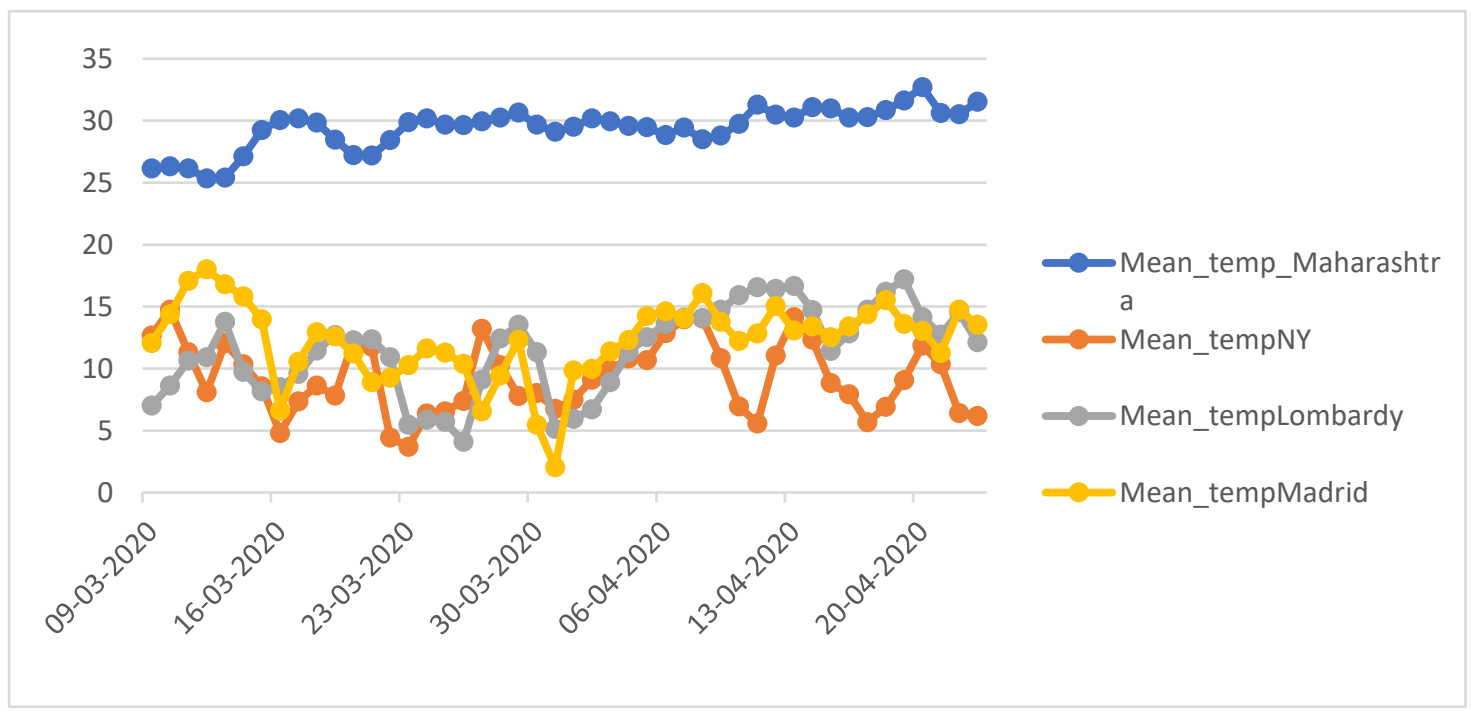

Figure 1 Graph of average mean temperature of the hourly data for all the four areas New York, Lombardy, Madrid and Maharashtra. 


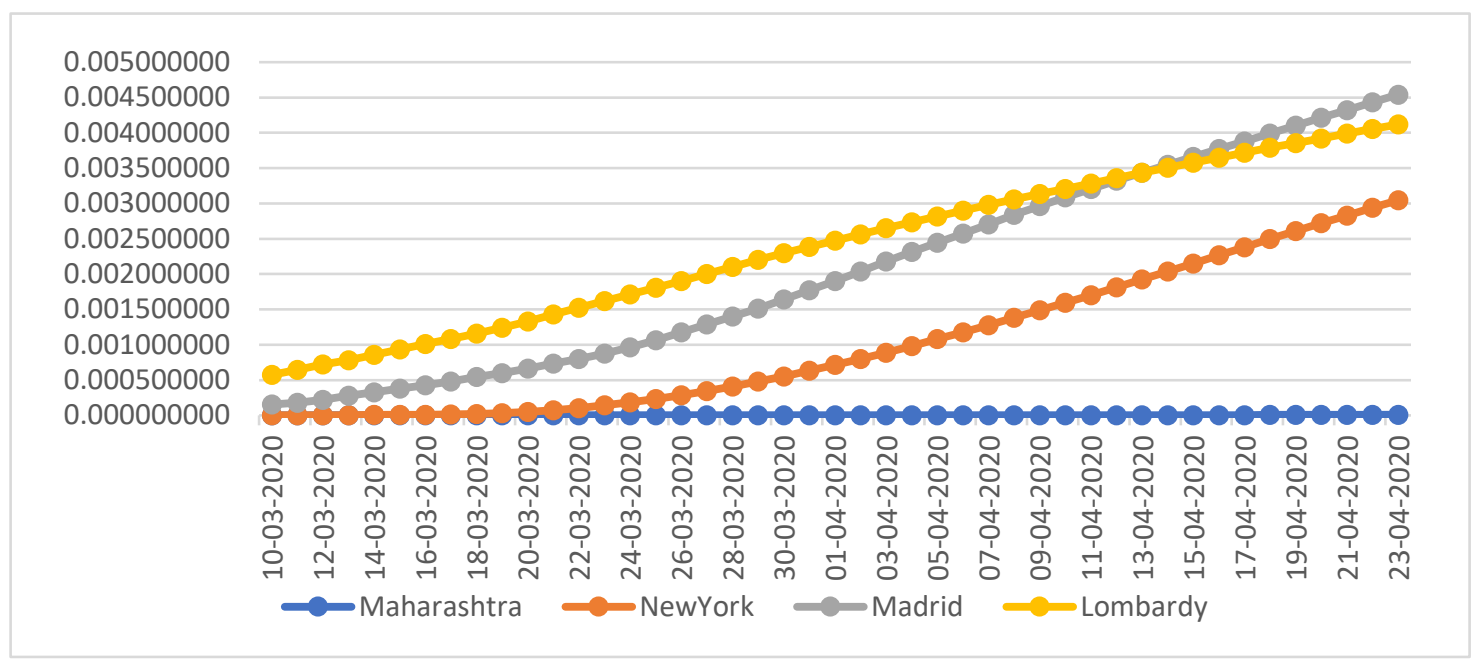

Figure 2 Graph of Cumulative mean of confirmed cases for all the four regions.

Having a deterministic view of above visualizations, the statistical analysis has been performed. In order to determine variance in number of confirmed cases and daily mean temperature for two parameters cumulative mean proportion of the population of confirmed cases and the average temperature for each specified region F- statistical analysis test as well as ANOVA for the unequal variance and t-test have been performed. The confidence level or alpha value of $95 \%$ has been taken into consideration in performing all the above-mentioned test. The Pearson's Correlation coefficient test has also been analysed to evaluate the impact or strength of these two quantitative variables.

Both the f-test and ANOVA help to observe the significant difference or variability around or within the population but the difference with the ANOVA is that it tells the combined variability of different groups from the overall or large distribution and to have precise information about the means of different groups multiple t-test with unequal variance has been performed. The multiple t-test would be the ad hoc test to let us know exactly which groups has difference in means.

The hypothesis that have been created for the statistical tests are:

$\mathrm{H}_{0}$ : - The variance of two groups are equal.

$\mathrm{H}_{1}$ : - The variance of two groups are not equal.

\section{Result and Discussion}

There is a significant negative correlation we'll able to establish between these two variables with the "Pearson Correlation" method

\section{Pearson's product-moment correlation}

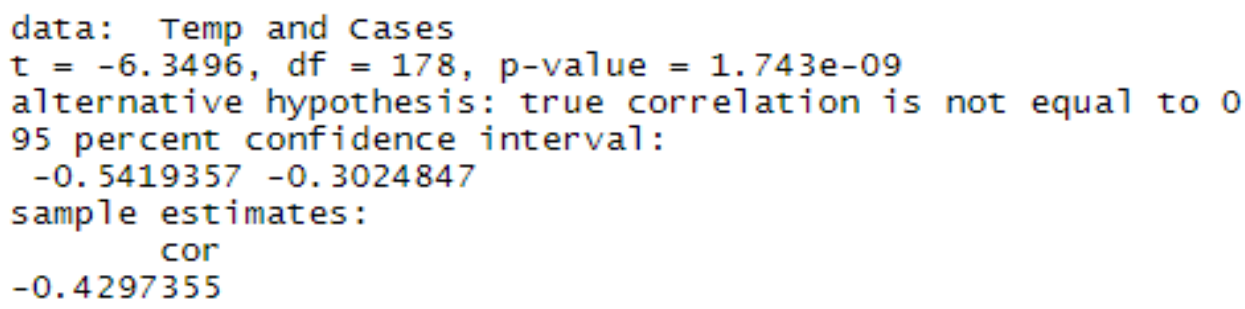


This shows that there is significant negative correlation between the two parameters as correlation values is -42 or approx. -43 which shows a strong negative correlation with $p$ value $1.743 \mathrm{e}-09$ less than the 0.05 significance level. Thus, increase in temperature leads to decrease in number of cases to have more specificity about the results further statistical analysis has been performed. F-test for

\section{Cumulative Confirmed Cases.}

Table 1

\begin{tabular}{|l|r|r|}
\hline \multicolumn{3}{|l|}{$\begin{array}{l}\text { F-Test Two-Sample for Variances } \\
\text { Madrid_Lombardy }\end{array}$} \\
\hline & Variable 1 & Variable 2 \\
\hline Mean & 0.002066 & 0.002403 \\
\hline Variance & $2.02 \mathrm{E}-06$ & $1.22 \mathrm{E}-06$ \\
\hline Observations & 45 & 45 \\
\hline df & 44 & 44 \\
\hline $\mathrm{F}$ & 1.660731 & \\
\hline $\begin{array}{l}\mathrm{P}(\mathrm{F}<=f) \text { one- } \\
\text { tail }\end{array}$ & 0.04805 & \\
\hline $\begin{array}{l}\text { F Critical one- } \\
\text { tail }\end{array}$ & 1.650935 & \\
\hline
\end{tabular}

Table 2

\begin{tabular}{|l|r|r|}
\hline \multicolumn{3}{|l|}{$\begin{array}{l}\text { F-Test Two-Sample for Variances } \\
\text { Madrid_NewYork }\end{array}$} \\
\hline & \multicolumn{1}{|l|}{ Variable 1 } & $\begin{array}{c}\text { Variable } \\
2\end{array}$ \\
\hline Mean & 0.00206597 & 0.00102 \\
\hline Variance & $2.02358 \mathrm{E}-06$ & $1 \mathrm{E}-06$ \\
\hline Observations & 45 & 45 \\
\hline df & 44 & 44 \\
\hline F & 2.014188241 & \\
\hline $\begin{array}{l}\mathrm{P}(\mathrm{F}<=\text { f) one- } \\
\text { tail }\end{array}$ & 0.01111628 & \\
\hline $\begin{array}{l}\text { F Critical one- } \\
\text { tail }\end{array}$ & 1.650934533 & \\
\hline
\end{tabular}

Table 3

Table 4

\begin{tabular}{|l|r|r|}
\hline \multicolumn{3}{|l|}{$\begin{array}{l}\text { F-Test Two-Sample for Variances } \\
\text { Madrid_Maharashtra }\end{array}$} \\
\hline & Variable 1 & $\begin{array}{c}\text { Variable } \\
2\end{array}$ \\
\hline Mean & 0.002066 & $1.99 \mathrm{E}-06$ \\
\hline Variance & $2.02 \mathrm{E}-06$ & $6.48 \mathrm{E}-12$ \\
\hline Observations & 45 & 45 \\
\hline df & 44 & 44 \\
\hline F & 312428.6 & \\
\hline $\begin{array}{l}\text { P(F<=f) one- } \\
\text { tail }\end{array}$ & $1.4 \mathrm{E}-109$ & \\
\hline $\begin{array}{l}\text { F Critical one- } \\
\text { tail }\end{array}$ & 1.650935 & \\
\hline
\end{tabular}

\begin{tabular}{|l|r|r|}
\hline \multicolumn{3}{|l|}{$\begin{array}{l}\text { F-Test Two-Sample for Variances } \\
\text { Lombardy_NewYork }\end{array}$} \\
\hline & Variable 1 & $\begin{array}{c}\text { Variable } \\
2\end{array}$ \\
\hline Mean & 0.002403 & 0.00102 \\
\hline Variance & $1.22 \mathrm{E}-06$ & $1 \mathrm{E}-06$ \\
\hline Observations & 45 & 45 \\
\hline df & 44 & 44 \\
\hline F & 1.212832 & \\
\hline $\begin{array}{l}\text { P(F<=f) one- } \\
\text { tail }\end{array}$ & 0.262435 & \\
\hline $\begin{array}{l}\text { F Critical one- } \\
\text { tail }\end{array}$ & 1.650935 & \\
\hline
\end{tabular}

Table 5

Table 6

\begin{tabular}{|l|r|r|}
\hline \multicolumn{3}{|l|}{$\begin{array}{l}\text { F-Test Two-Sample for Variances } \\
\text { Lombardy_Maharashtra }\end{array}$} \\
\hline & $\begin{array}{c}\text { Variable } \\
1\end{array}$ & Variable 2 \\
\hline Mean & 0.002403 & $1.99 \mathrm{E}-06$ \\
\hline Variance & $1.22 \mathrm{E}-06$ & $6.48 \mathrm{E}-12$ \\
\hline Observations & 45 & 45 \\
\hline df & 44 & 44 \\
\hline $\mathrm{F}$ & 188127.2 & \\
\hline $\begin{array}{l}\mathrm{P}(\mathrm{F}<=\mathrm{f}) \text { one- } \\
\text { tail }\end{array}$ & $9.6 \mathrm{E}-105$ & \\
\hline $\begin{array}{l}\text { F Critical one- } \\
\text { tail }\end{array}$ & 1.650935 & \\
\hline
\end{tabular}

\begin{tabular}{|l|r|r|}
\hline \multicolumn{3}{|l|}{$\begin{array}{l}\text { F-Test Two-Sample for Variances } \\
\text { NewYork_Maharashtra }\end{array}$} \\
\hline & \multicolumn{1}{l|}{ Variable 1 } & $\begin{array}{c}\text { Variable } \\
\end{array}$ \\
\hline Mean & 0.001020358 & $1.99 \mathrm{E}-06$ \\
\hline Variance & $1.00466 \mathrm{E}-06$ & $6.48 \mathrm{E}-12$ \\
\hline Observations & 45 & 45 \\
\hline df & 44 & 44 \\
\hline F & 155113.9184 & \\
\hline P(F<=f) one-tail & $6.7236 \mathrm{E}-103$ & \\
\hline F Critical one-tail & 1.650934533 & \\
\hline
\end{tabular}




\section{F-test for Mean temperature}

Table 7

\begin{tabular}{|l|r|r|}
\hline \multicolumn{3}{|l|}{$\begin{array}{l}\text { F-Test Two-Sample for Variances } \\
\text { Lombardy_Maharashtra }\end{array}$} \\
\hline & $\begin{array}{c}\text { Variable } \\
1\end{array}$ & $\begin{array}{c}\text { Variable } \\
2\end{array}$ \\
\hline Mean & 11.46205 & 29.40018 \\
\hline Variance & 12.34137 & 2.78051 \\
\hline Observations & 46 & 46 \\
\hline df & 45 & 45 \\
\hline F & 4.438528 & \\
\hline $\begin{array}{l}\text { P(F<=f) one- } \\
\text { tail }\end{array}$ & $9.35 \mathrm{E}-07$ & \\
\hline $\begin{array}{l}\text { F Critical one- } \\
\text { tail }\end{array}$ & 1.641516 & \\
\hline
\end{tabular}

Table 9 Table
Table 8

\begin{tabular}{|l|r|r|}
\hline \multicolumn{3}{|l|}{$\begin{array}{l}\text { F-Test Two-Sample for Variances } \\
\text { Lombardy_NY }\end{array}$} \\
\hline & Variable 1 & Variable 2 \\
\hline Mean & 11.46205 & 9.291395 \\
\hline Variance & 12.34137 & 8.192638 \\
\hline Observations & 46 & 46 \\
\hline df & 45 & 45 \\
\hline F & 1.506398 & \\
\hline $\begin{array}{l}\text { P(F<=f) one- } \\
\text { tail }\end{array}$ & 0.086611 & \\
\hline $\begin{array}{l}\text { F Critical one- } \\
\text { tail }\end{array}$ & 1.641516 & \\
\hline
\end{tabular}

10

\begin{tabular}{|l|r|r|}
\hline \multicolumn{3}{|l|}{$\begin{array}{l}\text { F-Test Two-Sample for Variances } \\
\text { Madrid_Maharashtra }\end{array}$} \\
\hline & Variable 1 & Variable 2 \\
\hline Mean & 12.26495 & 29.40018 \\
\hline Variance & 9.625608 & 2.78051 \\
\hline Observations & 46 & 46 \\
\hline df & 45 & 45 \\
\hline F & 3.461814 & \\
\hline P(F<=f) one-tail & $2.91 \mathrm{E}-05$ & \\
\hline $\begin{array}{l}\text { F Critical one- } \\
\text { tail }\end{array}$ & 1.641516 & \\
\hline
\end{tabular}

F-Test Two-Sample for Variances

Lombardy_Madrid

\begin{tabular}{|l|r|r|}
\hline & Variable 1 & Variable 2 \\
\hline Mean & 11.46205 & 12.26495 \\
\hline Variance & 12.34137 & 9.625608 \\
\hline Observations & 46 & 46 \\
\hline df & 45 & 45 \\
\hline $\mathrm{F}$ & 1.282139 & \\
\hline $\mathrm{P}(\mathrm{F}<=\mathrm{f})$ one-tail & 0.203858 & \\
\hline F Critical one-tail & 1.641516 & \\
\hline
\end{tabular}

Table 12

\begin{tabular}{|l|r|r|}
\hline \multicolumn{3}{|l|}{$\begin{array}{l}\text { F-Test Two-Sample for Variances } \\
\text { NY_Maharashtra }\end{array}$} \\
\hline & Variable 1 & Variable 2 \\
\hline Mean & 9.291395 & 29.40018 \\
\hline Variance & 8.192638 & 2.78051 \\
\hline Observations & 46 & 46 \\
\hline df & 45 & 45 \\
\hline F & 2.946451 & \\
\hline $\begin{array}{l}\text { P(F<=f) one- } \\
\text { tail }\end{array}$ & 0.000214 & \\
\hline $\begin{array}{l}\text { F Critical one- } \\
\text { tail }\end{array}$ & 1.641516 & \\
\hline
\end{tabular}

Table 11

\begin{tabular}{|l|r|r|}
\hline \multicolumn{3}{|l|}{$\begin{array}{l}\text { F-Test Two-Sample for Variances } \\
\text { Madrid_NY }\end{array}$} \\
\hline & Variable 1 & Variable 2 \\
\hline Mean & 12.26495 & 9.291395 \\
\hline Variance & 9.625608 & 8.192638 \\
\hline Observations & 46 & 46 \\
\hline df & 45 & 45 \\
\hline F & 1.17491 & \\
\hline P(F<=f) one-tail & 0.295507 & \\
\hline $\begin{array}{l}\text { F Critical one- } \\
\text { tail }\end{array}$ & 1.641516 & \\
\hline
\end{tabular}




\section{ANOVA Result for Confirmed cases}

To get the value for difference in each group " $t$-test" being performed and the results being attached below for each region "confirmed COVID-19 cases"

Table 13

\begin{tabular}{|l|r|r|}
\hline $\begin{array}{l}\text { t-Test: Two-Sample Assuming Unequal Variances } \\
\text { Madrid_Lombardy }\end{array}$ & \multicolumn{1}{l|}{ Variable 1 } & Variable 2 \\
\hline & 0.00206597 & 0.002403 \\
\hline Mean & $2.02358 \mathrm{E}-06$ & $1.22 \mathrm{E}-06$ \\
\hline Variance & 45 & 45 \\
\hline Observations & 0 & \\
\hline $\begin{array}{l}\text { Hypothesized Mean } \\
\text { Difference }\end{array}$ & 83 & \\
\hline df & -1.254023507 & \\
\hline t Stat & 0.106676931 & \\
\hline P(T<=t) one-tail & 1.663420175 & \\
\hline t Critical one-tail & 0.213353862 & \\
\hline P(T<=t) two-tail & 1.98895978 & \\
\hline t Critical two-tail & & \\
\hline
\end{tabular}

Table 15

\begin{tabular}{|l|r|r|}
\hline \multicolumn{3}{|l|}{$\begin{array}{l}\text { t-Test: Two-Sample Assuming Unequal Variances } \\
\text { Madrid_Maharashtra }\end{array}$} \\
\hline & Variable 1 & $\begin{array}{c}\text { Variable } \\
2\end{array}$ \\
\hline Mean & 0.002066 & $1.99 \mathrm{E}-06$ \\
\hline Variance & $2.02 \mathrm{E}-06$ & $6.48 \mathrm{E}-12$ \\
\hline Observations & 45 & 45 \\
\hline $\begin{array}{l}\text { Hypothesized Mean } \\
\text { Difference }\end{array}$ & 0 & \\
\hline df & 44 & \\
\hline t Stat & 9.73311 & \\
\hline P(T<=t) one-tail & $7.64 \mathrm{E}-13$ & \\
\hline t Critical one-tail & 1.68023 & \\
\hline P(T<=t) two-tail & $1.53 \mathrm{E}-12$ & \\
\hline t Critical two-tail & 2.015368 & \\
\hline
\end{tabular}

Table 14

t-Test: Two-Sample Assuming Unequal Variances Madrid_NY

\begin{tabular}{|l|r|r|}
\hline & \multicolumn{1}{|c|}{$\begin{array}{c}\text { Variable } \\
1\end{array}$} & $\begin{array}{c}\text { Variable } \\
2\end{array}$ \\
\hline Mean & 0.002066 & 0.00102 \\
\hline Variance & $2.02 \mathrm{E}-06$ & $1 \mathrm{E}-06$ \\
\hline Observations & 45 & 45 \\
\hline $\begin{array}{l}\text { Hypothesized Mean } \\
\text { Difference }\end{array}$ & 0 & \\
\hline $\mathrm{df}$ & 79 & \\
\hline $\mathrm{t}$ Stat & 4.030707 & \\
\hline $\mathrm{P}(\mathrm{T}<=\mathrm{t})$ one-tail & $6.36 \mathrm{E}-05$ & \\
\hline $\mathrm{t}$ Critical one-tail & 1.664371 & \\
\hline $\mathrm{P}(\mathrm{T}<=\mathrm{t})$ two-tail & 0.000127 & \\
\hline $\mathrm{t}$ Critical two-tail & 1.99045 & \\
\hline
\end{tabular}

Table 16

t-Test: Two-Sample Assuming Unequal Variances Lombardy_NY

\begin{tabular}{|l|r|r|} 
& & Variable \\
\multicolumn{1}{|c|}{ Mean } & 0.002402567 & 0.00102 \\
\hline Variance & $1.21849 \mathrm{E}-06$ & $1 \mathrm{E}-06$ \\
\hline Observations & 45 & 45 \\
\hline $\begin{array}{l}\text { Hypothesized Mean } \\
\text { Difference }\end{array}$ & 0 & \\
\hline $\mathrm{df}$ & 87 & \\
\hline $\mathrm{t}$ Stat & 6.21864032 & \\
\hline $\mathrm{P}(\mathrm{T}<=\mathrm{t})$ one-tail & $8.46725 \mathrm{E}-09$ & \\
\hline $\mathrm{t}$ Critical one-tail & 1.662557349 & \\
\hline $\mathrm{P}(\mathrm{T}<=\mathrm{t})$ two-tail & $1.69345 \mathrm{E}-08$ & \\
\hline $\mathrm{t}$ Critical two-tail & 1.987608282 & \\
\hline
\end{tabular}

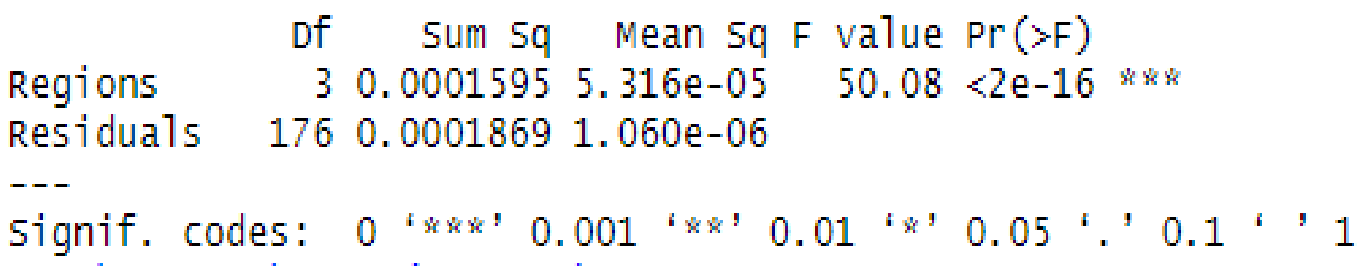

Figure 4 Analysis of Variance Result for all four regions of confirmed corona virus cases from $10^{\text {th }}$ March 2020 till $23^{\text {rd }}$ April 2020. Variance is quite significant among some groups as F value is 50.08 with significant $p$ value less than the 0.05 . 
Table 17

t-Test: Two-Sample Assuming Unequal Variances Lombardy_Maharashtra

\begin{tabular}{|l|r|r|}
\hline & Variable 1 & \multicolumn{1}{|c|}{\begin{tabular}{c} 
Variable \\
\hline Mean
\end{tabular}} \\
\hline Variance & 0.002403 & $1.99 \mathrm{E}-06$ \\
\hline Observations & 45 & 45 \\
\hline $\begin{array}{l}\text { Hypothesized Mean } \\
\text { Difference }\end{array}$ & 0 & \\
\hline df & 44 & \\
\hline t Stat & 14.58851 & \\
\hline $\mathrm{P}(\mathrm{T}<=\mathrm{t})$ one-tail & $9.11 \mathrm{E}-19$ & \\
\hline $\mathrm{t}$ Critical one-tail & 1.68023 & \\
\hline $\mathrm{P}(\mathrm{T}<=\mathrm{t})$ two-tail & $1.82 \mathrm{E}-18$ & \\
\hline $\mathrm{t}$ Critical two-tail & 2.015368 & \\
\hline
\end{tabular}

Table 18

\begin{tabular}{|l|r|r|}
\hline \multicolumn{3}{|l|}{ t Test: Two-Sample Assuming Unequal } \\
Variances NY_Maharshtra \\
\hline & Variable 1 & $\begin{array}{c}\text { Variable } \\
2\end{array}$ \\
\hline Mean & 0.00102 & $1.99 \mathrm{E}-06$ \\
\hline Variance & $1 \mathrm{E}-06$ & $6.48 \mathrm{E}-12$ \\
\hline Observations & 45 & 45 \\
\hline $\begin{array}{l}\text { Hypothesized Mean } \\
\text { Difference }\end{array}$ & 0 & \\
\hline df & 44 & \\
\hline $\mathrm{t}$ Stat & 6.815555 & \\
\hline $\mathrm{P}(\mathrm{T}<=\mathrm{t})$ one-tail & $1.07 \mathrm{E}-08$ & \\
\hline $\mathrm{t}$ Critical one-tail & 1.68023 & \\
\hline $\mathrm{P}(\mathrm{T}<=\mathrm{t})$ two-tail & $2.13 \mathrm{E}-08$ & \\
\hline $\mathrm{t}$ Critical two-tail & 2.015368 & \\
\hline
\end{tabular}

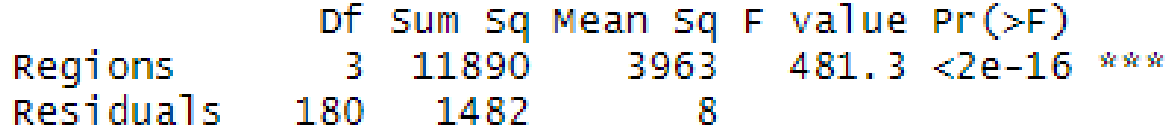

---
signif. codes:
0 ' 'sk'
0.001 ' $* *$,
0.01 '*
0.05 '. 0.1 ', 1

Figure 5 Analysis of Variance for mean temperature for the four regions with F-value 481.3 and $p$ value less than significant level which is 0.05 thus it indicates that variance in group of means is present in the data and this is not random by chance.

\section{ANOVA Result for mean Temperature}

For significant difference for each region "average temperature t-test" being performed and results being attached below

Table 19

\begin{tabular}{|l|r|r|}
\hline t-Test: Two-Sample Assuming Unequal Variances \\
\hline & Mean_tempNY & $\begin{array}{c}\text { Mean_temp } \\
\text { Maharashtra }\end{array}$ \\
\hline Mean & 9.291395 & 29.40018 \\
\hline Variance & 8.192638 & 2.78051 \\
\hline Observations & 46 & 46 \\
\hline $\begin{array}{l}\text { Hypothesized } \\
\text { Mean } \\
\text { Difference }\end{array}$ & 0 & \\
\hline df & 72 & \\
\hline t Stat & -41.1717 & \\
\hline P(T<=t) one-tail & $4.39 \mathrm{E}-52$ & \\
\hline $\begin{array}{l}\text { t Critical one- } \\
\text { tail }\end{array}$ & 1.666294 & \\
\hline P(T<=t) two-tail & $8.77 \mathrm{E}-52$ & \\
\hline $\begin{array}{l}\text { t Critical two- } \\
\text { tail }\end{array}$ & 1.993464 & \\
\hline
\end{tabular}


Table 20

\begin{tabular}{|l|r|r|}
\hline t-Test: Two-Sample Assuming Unequal Variances \\
\hline & $\begin{array}{c}\text { Mean_temp } \\
\text { Madrid }\end{array}$ & $\begin{array}{c}\text { Mean_temp } \\
\text { NY }\end{array}$ \\
\hline Mean & 12.26495 & 9.291395 \\
\hline Variance & 9.625608 & 8.192638 \\
\hline Observations & 46 & 46 \\
\hline $\begin{array}{l}\text { Hypothesized } \\
\text { Mean Difference }\end{array}$ & 0 & \\
\hline df & 89 & \\
\hline t Stat & 4.777732 & \\
\hline P(T<=t) one-tail & $3.47 \mathrm{E}-06$ & \\
\hline t Critical one-tail & 1.662155 & \\
\hline P(T<=t) two-tail & $6.95 \mathrm{E}-06$ & \\
\hline t Critical two-tail & 1.986979 & \\
\hline
\end{tabular}

Table 22

\begin{tabular}{|l|r|r|}
\hline \multicolumn{3}{|c|}{ t-Test: Two-Sample Assuming Unequal Variances } \\
\hline & $\begin{array}{c}\text { Mean_temp } \\
\text { Lombardy }\end{array}$ & $\begin{array}{c}\text { Mean_temp_ } \\
\text { Maharashtra }\end{array}$ \\
\hline Mean & 11.46205 & 29.40018 \\
\hline Variance & 12.34137 & 2.78051 \\
\hline Observations & 46 & 46 \\
\hline $\begin{array}{l}\text { Hypothesized } \\
\text { Mean Difference }\end{array}$ & 0 & \\
\hline df & 64 & \\
\hline t Stat & -31.2862 & \\
\hline P(T<=t) one-tail & $8.4 \mathrm{E}-41$ & \\
\hline t Critical one-tail & 1.669013 & \\
\hline P(T<=t) two-tail & $1.68 \mathrm{E}-40$ & \\
\hline t Critical two-tail & 1.99773 & \\
\hline
\end{tabular}

Table 21

\begin{tabular}{|l|r|r|}
\hline t-Test: Two-Sample Assuming Unequal Variances \\
\hline & $\begin{array}{c}\text { Mean_temp } \\
\text { Lombardy }\end{array}$ & $\begin{array}{c}\text { Mean_temp } \\
\text { Madrid }\end{array}$ \\
\hline Mean & 11.46205 & 12.26495 \\
\hline Variance & 12.34137 & 9.625608 \\
\hline Observations & 46 & 46 \\
\hline $\begin{array}{l}\text { Hypothesized } \\
\text { Mean Difference }\end{array}$ & 0 & \\
\hline df & 89 & \\
\hline t Stat & -1.16186 & \\
\hline P(T<=t) one-tail & 0.124199 & \\
\hline t Critical one-tail & 1.662155 & \\
\hline P(T<=t) two-tail & 0.248398 & \\
\hline t Critical two-tail & 1.986979 & \\
\hline
\end{tabular}

Table 23

\begin{tabular}{|l|r|r|}
\hline t-Test: Two-Sample Assuming Unequal Variances \\
\hline & $\begin{array}{c}\text { Mean_temp } \\
\text { Madrid }\end{array}$ & $\begin{array}{c}\text { Mean_temp_ } \\
\text { Maharashtra }\end{array}$ \\
\hline Mean & 12.26495 & 29.40018 \\
\hline Variance & 9.625608 & 2.78051 \\
\hline Observations & 46 & 46 \\
\hline Hypothesized & 0 & \\
Mean Difference & 69 & \\
\hline df & -32.9952 & \\
\hline t Stat & $2.75 \mathrm{E}-44$ & \\
\hline P(T<=t) one-tail & 1.667239 & \\
\hline t Critical one-tail & $5.5 \mathrm{E}-44$ & \\
\hline P(T<=t) two-tail & 1.994945 & \\
\hline t Critical two-tail & & \\
\hline
\end{tabular}


Table 24

\begin{tabular}{|l|r|r|}
\hline t-Test: Two-Sample Assuming Unequal Variances \\
\hline & $\begin{array}{c}\text { Mean_temp } \\
\text { Lombardy }\end{array}$ & $\begin{array}{c}\text { Mean_temp } \\
\text { NY }\end{array}$ \\
\hline Mean & 11.46205 & 9.291395 \\
\hline Variance & 12.34137 & 8.192638 \\
\hline Observations & 46 & 46 \\
\hline $\begin{array}{l}\text { Hypothesized } \\
\text { Mean } \\
\text { Difference }\end{array}$ & 0 & \\
\hline df & 86 & \\
\hline t Stat & 3.24887 & \\
\hline P(T<=t) one-tail & 0.000827 & \\
\hline $\begin{array}{l}\text { t Critical one- } \\
\text { tail }\end{array}$ & 1.662765 & \\
\hline $\begin{array}{l}\text { P(T<=t) two- } \\
\text { tail }\end{array}$ & 0.001654 & \\
\hline $\begin{array}{l}\text { t Critical two- } \\
\text { tail }\end{array}$ & 1.987934 & \\
\hline
\end{tabular}

With all the above results we'll able to conclude that maximum variance is seen for both the parameters, while comparing Maharashtra region with all three i.e. Lombardy, Madrid and New York City as f-value is larger than the f-critical value and p-vale is smaller than the significance level which is 0.05 thus, we'll fail to accept the null hypothesis. But in case of Lombardy and New York number of variances for cumulative coronavirus confirmed cases is equal, as the f-value 1.212832 which is comparatively smaller to the f-critical value (1.650935) and p-value i.e. 0.262435 greater than significance value 0.05 in this case we'll fail to reject the null hypothesis and same conclusion goes for mean temperature variable where $\mathrm{f}$-value is 1.50 which is lower than the f-critical range (1.64) and p-value is 0.086 which is way above higher than the significance level. Madrid and Lombardy do have a special case where we observe a slight variance between the values of cumulative confirmed cases as f-value (1.660731) is slightly more than the f-critical one (1.650) and $p$ value (0.04805) i.e. approximately equal to 0.05 and equal variance is able to notice in the temperature parameter. Madrid and New York have unequal variance in number of confirmed cases but have equal variance for mean temperature.

Further, to determine whether the difference is significant t-test has been done, except for Madrid and Lombardy where the two tailed p- value $(0.213353862)$ is higher than the 0.05 for the confirmed cases parameter and same goes for the mean temperature one ( $p$-value 0.248398 ) the difference is not significant but for rest of the regions the difference is quite significant as p-value is smaller than the significance level for both i.e. cumulative confirmed COVID-19 cases and mean temperature.

\section{Conclusion}

Thus, we'll able to infer that environmental factor "temperature" is playing quite a substantial role in daily rate of confirmed cases as the above statistical analysis that includes Pearson's correlation, $\mathrm{f}$ statistics, ANOVA and t-test for unequal variances shows that daily rate of confirmed cases are higher in the areas with lower temperature even the correlation also gives a strong evidence that there is high negative correlation between mean temperature and cumulative number of confirmed cases. 


\section{References}

Chan KH, Peiris JS, Lam SY, Poon LL, Yuen KY, Seto WH. The effects of temperature and relative humidity on the viability of the SARS coronavirus. Advances in virology. 2011;2011.

Chambers, J. M. and Hastie, T. J. (1992) Statistical Models in S, Wadsworth \& Brooks/Cole.

D. J. Best \& D. E. Roberts (1975). Algorithm AS 89: The Upper Tail Probabilities of Spearman's rho. Applied Statistics, 24, 377-379. doi: 10.2307/2347111.

Myles Hollander \& Douglas A. Wolfe (1973), Nonparametric Statistical Methods.New York: John Wiley \& Sons. Pages 185-194 (Kendall and Spearman tests).

WHO, Coronavirus disease (COVID-2019) situation reports, Available online:

https://www.who.int/emergencies/diseases/novel-coronavirus-2019/situation-reports 